\title{
Evolución del desarrollo del color en sistema modelo de composición similar al dulce de leche. Influencia del tiempo de calentamiento y del pH
}

\author{
Color development in dulce de leche-like system. \\ Effect of heating time and $\mathrm{pH}$
}

\author{
Rodríguez, Analía (1), Piagentini, Andrea (2), Rozycki, Sergio ${ }^{(2)}$, Lema, Patricia ${ }^{(3)}$, Pauletti, Miguel S. (2), Panizzolo, Luis \\ Alberto $^{(1)}$ \\ (1) Cátedra de Ciencia y Tecnología de los Alimentos, Facultad de Química, Universidad de la República, Uruguay - \\ ${ }^{(2)}$ Instituto de Tecnología de Alimentos (FIQ-UNL), Santa Fe, Argentina - ${ }^{(3)}$ Instituto de Química, Facultad de \\ Ingeniería, Universidad de la República. Uruguay \\ Contacto: arodriguez@fq.edu.uy
}

Recibido: 15/6/2012 - Aprobado: 17/11/2012

\section{Resumen}

El color es un atributo importante en la elección del producto dulce de leche por parte del consumidor. El mismo se desarrolla en el proceso de elaboración debido principalmente a las reacciones de pardeamiento no enzimático, las cuales se ven influenciadas por varios factores. En este trabajo se evaluó el desarrollo de color, con el tiempo de tratamiento térmico y el pH inicial como variables tecnológicas, en un sistema modelo compuesto de caseinato, lactosa, sacarosa y agua destilada cuya composición fue similar a la del dulce de leche comercial. El estudio del color se realizó utilizando los parámetros de medición de color del sistema CIELAB y el índice de Kubelka Munk (K/S). Con ambos métodos se constató un aumento significativo del dE*ab y del K/S con el tiempo de tratamiento térmico y el pH inicial. Con el sistema CIELAB además se observó una tendencia a disminuir la luminosidad con el tiempo y el aumento del $\mathrm{pH}$ inicial, y el aumento de a* con el tiempo.

Palabras clave: Dulce de leche, maillard, color, melanoidinas.

\begin{abstract}
$\underline{\text { Abstract }}$
Color in dulce de leche is an important product attribute in consumers choice. Color development is mainly due to non-enzymatic browning reactions during the manufacture, which are influenced by several factors. In this work color development and the influence of technological variables, time of heat treatment and initial $\mathbf{p H}$, were evaluated on a model system consisting of caseinate, lactose, sucrose and distilled water, which composition was similar to commercial dulce de leche. Color was studied using color parameters, the CIELAB system and the Kubelka Munk índex (K/S). Both methods showed a significant increase in $\mathrm{dE}^{*} \mathrm{ab}$ and $\mathrm{K} / \mathrm{S}$ with heat treatment time and initial $\mathrm{pH}$. The CIELAB system also showed a Luminance decreasement with time and initial $\mathrm{pH}$ increase, and $\mathrm{a}^{*}$ increase with time.

Keywords: dulce de leche, maillard, color, melanoidins.
\end{abstract}

\section{Introducción}

El dulce de leche es el producto obtenido por el calentamiento de leche adicionada de sacarosa y su concentración (en algunos casos se remplaza parcialmente por glucosa, fructosa u otro azúcar permitido según normativa vigente en el país donde se elabore), el cual adquiere coloración, consistencia y sabor característicos como consecuencia de las reacciones de pardeamiento no enzimático que ocurren, con la reacción de Maillard como la principal responsable de las transformaciones (Malec et al., 1999).

La reacción de Maillard es la descripción general de una serie de reacciones complejas debidas a la reacción de grupos amino libres como aminas, aminoácidos, péptidos y proteínas con compuesto carbonilo, particularmente azúcares reductores. Es especialmente importante en sistemas alimentarios en los que los productos de la reacción pueden ser responsables del aroma, gusto y apariencia de los alimentos (Fayle y Gerrard, 2005), entre ellos el color. El color pardo característico del dulce de leche es el resultante del desarrollo de los compuestos coloreados denominados melanoidinas, que imparten un color marrón oscuro (pardo) en las etapas finales de la reacción de Maillard. Esta reacción es afectada por varios factores, como el pH (Petriella et al., 1985; Hayashi y Namiki, 1986; Pauletti et al., 1999; Davies y Labuza, 2003; Rozycki, 2003; Nursten, 2005; Dattatreya y Rankin, 2006;) y la temperatura (Pauletti et al., 1999; Nursten, 2005; Rozycki et al., 2007), entre otros (Davies y Labuza, 2003).

Considerando que en las etapas avanzadas de la reacción de Maillard se forman las melanoidinas, pigmentos pardos de elevado peso molecular (Ferrer et al., 1999), la medida de color brinda un índice útil para evaluar la intensidad de esta reacción (Jiménez et al., 2003), y puede ser un indicador no específico.

El color en el dulce de leche juega un rol preponderante en la evaluación de la calidad comercial y aceptación por parte de los consumidores. Hough et al. $(1986 ; 1992)$ encontraron que para el consumidor el color y la textura eran más importantes que el sabor. En cuanto al color, las preferencias del mercado no son uniformes, hecho que se refleja en la gama de colores que allí se oferta (Pauletti, 2001). Sin embargo, a nivel industrial es de gran importancia mantener la 
uniformidad del color (Hough et al., 1991), para lo cual se presenta la problemática de encontrar la metodología más adecuada para la medición del color en el dulce de leche. Son comúnmente usados los métodos de evaluación visual (subjetivos) y el uso de estándares de color, aunque la medida objetiva del color por métodos instrumentales es deseada y aplicada ampliamente en la industria de alimentos (Wrolstad y Smith, 2010).

El color se puede definir como la sensación que experimenta el observador cuando la energía radiante del espectro visible (380$770 \mathrm{~nm}$ ) alcanza la retina del ojo. Dentro de los métodos instrumentales de medición de color se halla el colorímetro, con el cual se puede medir cuánto se desvían parámetros del color de una muestra frente a un estándar, o cambios de batch a batch, cambios por proceso o almacenamiento, etcétera. Las diferencias de color son calculadas como la diferencia de $\mathrm{L}^{*} \mathrm{a} \mathrm{b}^{*}$ entre muestras, y es posible hacer las siguientes interpretaciones: $\mathrm{dL}^{*}$ positivo significa mayor luminosidad de la muestra vs. estándar y viceversa; da* positivo significa más 'rojo' o menos 'verde' y viceversa; db* positivo mas 'amarillo' o menos 'azul' y viceversa. Cuando se desea conocer si hay diferencias de color entre dos muestras y su magnitud, sin importar la dirección, se puede calcular la diferencia total de color $\mathrm{dE}^{*} \mathrm{ab}=\left(\mathrm{dL}^{* 2}+\mathrm{da}^{* 2}+\right.$ $\left.\mathrm{db}^{* 2}\right)^{1 / 2}$ (Wrolstad y Smith, 2010).

En el caso de alimentos translúcidos (no son totalmente opacos ni completamente transparentes), como lo es el dulce de leche, para detectar correctamente diferencias entre muestras (tanto visuales como instrumentales) resulta influyente la forma de presentación de la muestra. Los problemas pueden solucionarse aplicando el análisis de capa fina de Kubelka-Munk, por el cual se mide la reflexión en una muestra de poco espesor $(4-5 \mathrm{~mm})$ utilizando un fondo blanco y uno negro, relacionándolo con un coeficiente de absorción $(\mathrm{K})$ y otro de dispersión (S), según:

$$
\frac{K}{S}=\frac{(1-R \infty)^{2}}{2 R \infty}
$$

$\mathrm{R}_{\infty}=$ reflexión de una capa tan gruesa, llamada de espesor infinito, que al aumentar el espesor no varía la reflexión (Calvo y Durán, 1997).

El parámetro $\mathrm{K}$ está relacionado con la cantidad de grupos cromóforos presentes (Pauletti, 1999; Rozycki, 2003). El índice de Kubelka Munk ha sido ampliamente utilizado en medidas de color en dulce de leche o sistemas modelos representativos (Hough et al., 1991; Pauletti, 1999; Rozycki, 2003, 2007, 2010), por haberse demostrado que es representativo de la percepción sensorial (Buera et al., 1990).

Como fue mencionado anteriormente, la reacción de Maillard es afectada por varios parámetros, entre ellos el $\mathrm{pH}$, debido a que varias de las etapas de la reacción son catalizadas por mecanismos ácidobasico. En la primera etapa de la reacción, la condensación aminocarbonilo, el medio ácido aumenta la polaridad del grupo carbonilo y el medio básico aumenta el carácter nucleofílico del grupo amino, el cual en condiciones ácidas no tiene participación activa porque pierde el par de electrones libres (Dattatreya y Rankin, 2006). Posteriormente, la degradación del compuesto de Amadori formado también es influenciada por el $\mathrm{pH}$. A pH ácidos se ve favorecida la ruta 1,2 enolización con la formación de furfurales, mientras que a $\mathrm{pH}$ básico se ve favorecida la vía de 2,3 enolización en la que se producen compuestos de seis carbonos y otras reductonas (Delgado, 1993; Nursten, 2005; Dattatreya y Rankin, 2006). La etapa de fragmentación de los azúcares, en la que se producen intermediarios de dos y tres carbonos muy reactivos, es altamente dependiente del $\mathrm{pH}$. Hay gran formación de compuestos de fragmentación a $\mathrm{pH}$ básico y poca a $\mathrm{pH}$ ácido. Es al pH alcalino al cual se da mayor desarrollo de color y al cual se favorece la vía de la fragmentación (Hayashi y Namiki, 1986). El pH también afecta los compuestos pardos formados. Hayashi y Namiki (1985) encontraron que las melanoidinas formadas a $\mathrm{pH}$ ácido y alcalino son diferentes al estudiar la relación nitrógeno/carbono (N/C) de las mismas formadas a partir de mezclas equimolares de glucosa y $\beta$-alanina a diferentes $\mathrm{pH}$. Observaron que esta relación aumentaba significativamente con el aumento del $\mathrm{pH}$ inicial, dado que en esas condiciones está favorecida la vía de fragmentación de azúcares y el desarrollo de color.

El objetivo de este trabajo fue evaluar para un sistema lácteo de alto contenido de sacarosa la influencia del pH inicial $(6 ; 6,8 ; 7,5)$ y el tiempo de calentamiento $(0,10,30,60$ minutos) sobre el desarrollo del color, analizando cada componente del color del sistema CIELAB y el índice de Kubelka Munk (K/S).

\section{Materiales y Métodos}

\section{Preparación del sistema modelo}

Se preparó a partir de caseinato de sodio (LACTOPROT Deutchland GMBH, $95 \%$ pureza), $\alpha$-lactosa (Sigma, Netherland), sacarosa comercial (azúcar blanco refinado ALUR, Uruguay) y agua destilada, en la proporción en que se encuentran en un dulce de leche comercial (7 \% proteína; $11 \%$ lactosa; $30 \%$ agua y $44 \%$ sacarosa), obteniéndose una aw final de 0,85 , dentro del rango encontrado para el producto (Tamime, 2009; Castañeda et al., 2004; Demiate et al., 2001). Los sólidos se mezclaron en seco y se agregó el agua a $80^{\circ} \mathrm{C}$ con fuerte agitación hasta disolución de los sólidos, y posteriormente se homogenizó a $18000 \mathrm{rpm}$ por 3 minutos en homogeneizador Ultraturrax T25 (IKA-Labortechnik) con dispersante S25N-25F. El pH se midió potenciométricamente con peachímetro (Orion Research digital ionalyzer/501) y se ajustó al valor de diseño ( $\mathrm{pH} \mathrm{6}$; $6,8 ; 7,5)$ utilizando ácido láctico o hidróxido de sodio según el caso, homogeneizando mediante agitación mecánica manual y magnética y, finalmente, con Ultraturrax.

\section{Diseño experimental}

El diseño consistió en Unidades experimentales (tubos cerrados) que contenían el sistema modelo, dispuestas al azar en una gradilla, que fueron sumergidas en un baño de aceite a la temperatura preestablecida, constante e igual a $112{ }^{\circ} \mathrm{C}$ (con agitación y control automático de temperatura), utilizando una plancha calefactora (IKA C-MAG HS7) y termocupla (IKA ETS-D5). Las muestras fueron retiradas a los tiempos seleccionados $(0,10,30,60$ minutos) y enfriadas inmediatamente con agua helada $\left(2^{\circ} \mathrm{C}\right)$. Las experiencias se realizaron por duplicado.

\section{Evaluación del color}

Las medidas de color se realizaron en un colorímetro (Konica Minolta $600 \mathrm{~d}$ ) con iluminante D65, $10^{\circ}$ de ángulo del observador y componente especular excluido (Dattatreya y Ranking, 2006).

Se utilizaron celdas no estándares de color blanco mate y negro mate de $28 \mathrm{~mm}$ diámetro y $4 \mathrm{~mm}$ altura. Para obtener el índice de Kubelka Munk se midió la reflectancia a 450, 560 y $685 \mathrm{~nm}$, y se utilizó el software SBRT (Shelf Backing Reflectance Transformation Software), apropiado para pequeñas muestras (Pauletti et al., 1999; Rozycki et al., 2010). Para el sistema CIELAB (parámetros L*, a, $\mathrm{b}^{*}$ ) se tomaron los datos obtenidos con la celda blanca (Dattatreya y Ranking, 2006) y se calculó la diferencia de color con la ecuación $\mathrm{dE}^{*} \mathrm{ab}=\left(\mathrm{dL}^{* 2}+\mathrm{da}^{* 2}+\mathrm{db}^{* 2}\right)^{1 / 2}$, tomando como estándar o referencia a la muestra a tiempo cero. Todas las mediciones se realizaron por triplicado.

\section{Análisis Estadístico}

El tratamiento estadístico de datos se realizó mediante el método de ANOVA, con $\alpha \leq 0,05$, y en el caso de diferencias significativas se realizó la comparación de medias por el método de Tukey, con el programa Infostat/L versión libre, 2011. 


\section{Resultados}

En la Figura 1 se muestra la evolución con el tiempo de reacción de la diferencia de color $\left(\mathrm{dE}^{*} \mathrm{ab}\right)$ respecto a la muestra a tiempo cero, para los $\mathrm{pH}$ iniciales de estudio.

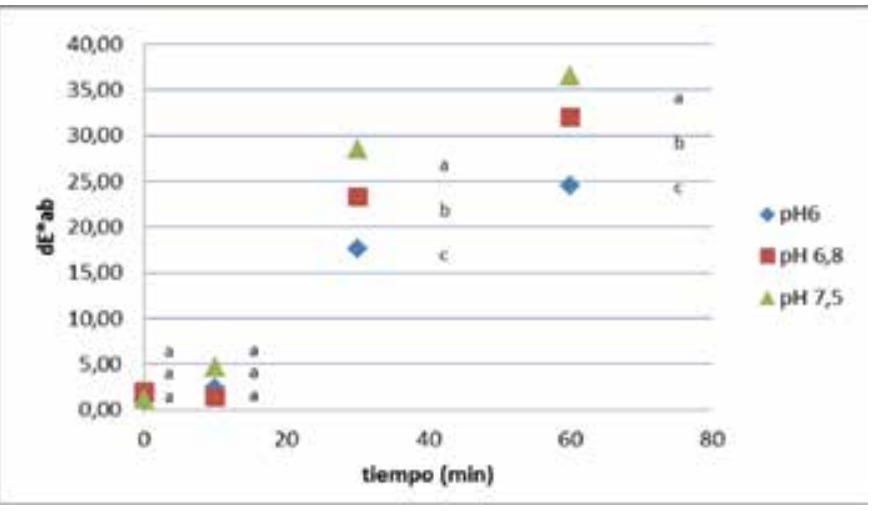

Figura 1. Evolución de $d^{\star}{ }^{\star} a b$ vs. tiempo de tratamiento térmico para los $3 \mathrm{pH}$ iniciales (medias con una letra común no son significativamente diferentes para cada tiempo, $\alpha \leq 0,05$ ).

Se observó un rápido aumento de color con el tiempo a partir de la muestra retirada a los 30 minutos; el mayor desarrollo de color se produce a $\mathrm{pH}$ inicial 7,5, seguido del $\mathrm{pH}$ inicial 6,8 y por último el $\mathrm{pH}$ inicial 6.

En la Figura 2 se observa la evolución del color medida con el índice de Kubelka Munk. Ambas funciones del color, dE*ab y K/S, presentaron un comportamiento similar, con un periodo de inducción de corta duración seguido de un aumento rápido de los parámetros. El período de inducción puede ser explicado por el hecho de que los compuestos coloreados en las reacciones de pardeamiento no enzimático se desarrollan en las últimas etapas (Nursten, 2005; Ferrer et al., 1999).

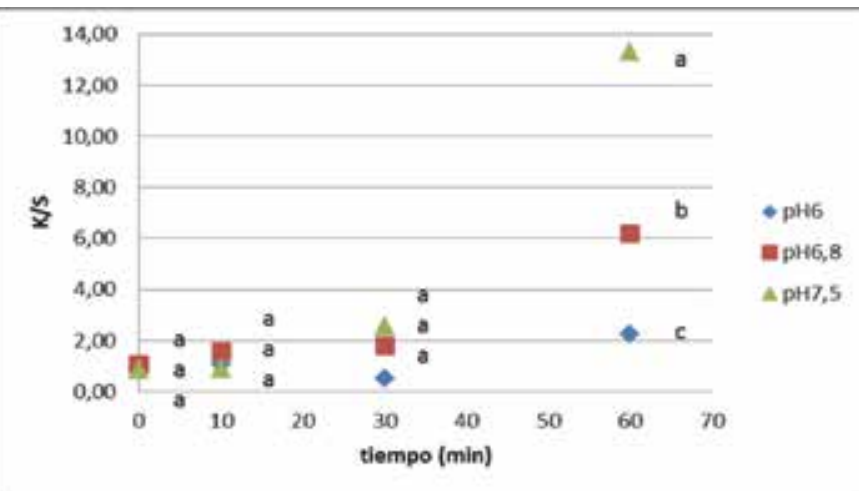

Figura 2. Evolución de K/S vs.tiempo de tratamiento térmico para los $3 \mathrm{pH}$ iniciales (medias con una letra común no son significativamente diferentes para cada tiempo, $\alpha \leq 0,05)$.

Se observó que el K/S, a 30 minutos de calentamiento, tiene un valor cercano al de las primeras etapas de calentamiento, mientras que los valores a 60 minutos fueron mayores a los anteriores; en el caso de $\mathrm{dE}^{*}$ ab a 30 minutos presentó valores significativamente mayores a los de las primeras etapas y similares a los correspondientes a las etapas finales de calentamiento (60 minutos). Con ambos métodos se pudo observar diferencias significativas en el desarrollo de color entre las diferentes condiciones de $\mathrm{pH}$ inicial, que aumentaron a medida que esta variable fue mayor, siendo una influencia muy relevante.
Los pH alcalinos aceleran la reacción de Maillard (Badui, 1990), lo cual fue observado para sistemas acuosos de azúcar-aminoácidos por el aumento de la tasa de pardeamiento (Kato et al., 1969; Wolfrom et al., 1974; Ashoor \& Zent, 1984). Petriella et al. (1985) observaron que los valores de energía de activación de la reacción de Maillard varían según el pH. Pauletti et al. (1999) y Rozycki (2003), en estudios de la influencia de parámetros en la cinética del color (utilizando sistemas de composición similar al dulce de leche a partir de leche en polvo, sacarosa y agua destilada), encontraron influencia directa del $\mathrm{pH}$ en la cinética. Ambos parámetros, calculados en este trabajo, reflejan apropiadamente los cambios de color y resultarían muy útiles para cuantificarlo incluso a nivel industrial, lo cual permitiría el control de calidad del producto y el diseño adecuado de equipos, principalmente en el método continuo de producción de dulce de leche.

En el análisis de los parámetros del color, con respecto a la luminosidad $\left(\mathrm{L}^{*}\right)$ se registró con el tiempo de tratamiento térmico valores de $\mathrm{dL}^{*}$ negativo (Figura 3 ), lo que significa menor luminosidad o mayor tendencia al negro de la muestra con respecto a la referencia (muestra a tiempo cero).

Con respecto al $\mathrm{pH}$, hay mayor disminución de la luminosidad $\left(\mathrm{L}^{*}\right)$ a mayor $\mathrm{pH}$ inicial.

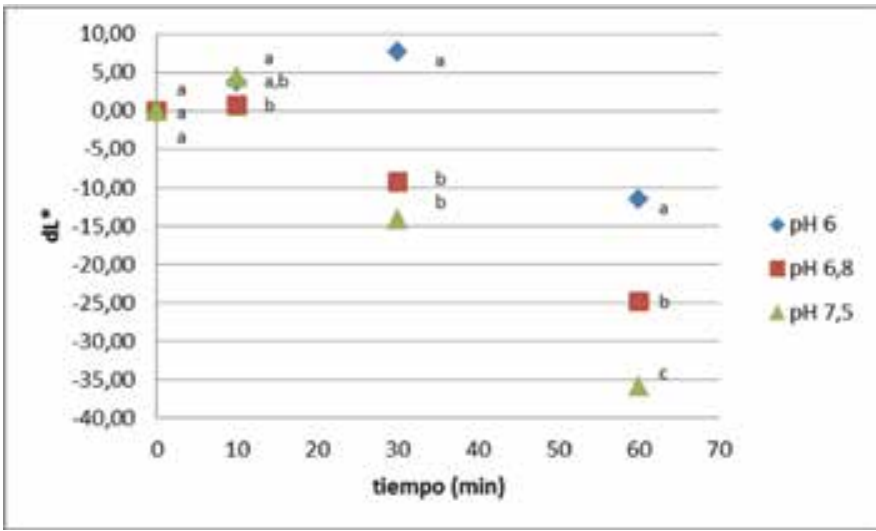

Figura 3. Diferencia de luminosidad vs. tiempo de tratamiento térmico para los $3 \mathrm{pH}$ iniciales (medias con una letra común no son significativamente diferentes para cada tiempo, $\alpha \leq 0,05$ ).

En cuanto a la evolución del parámetro del color a* (Figura 4), se vio un aumento con el tiempo de tratamiento térmico y mayor da* (colores mas rojos) a mayor $\mathrm{pH}$ en las primeras etapas de calentamiento (tiempos menores), sin que se hallaran diferencias significativas en el tiempo final para los tres $\mathrm{pH}$ ensayados.

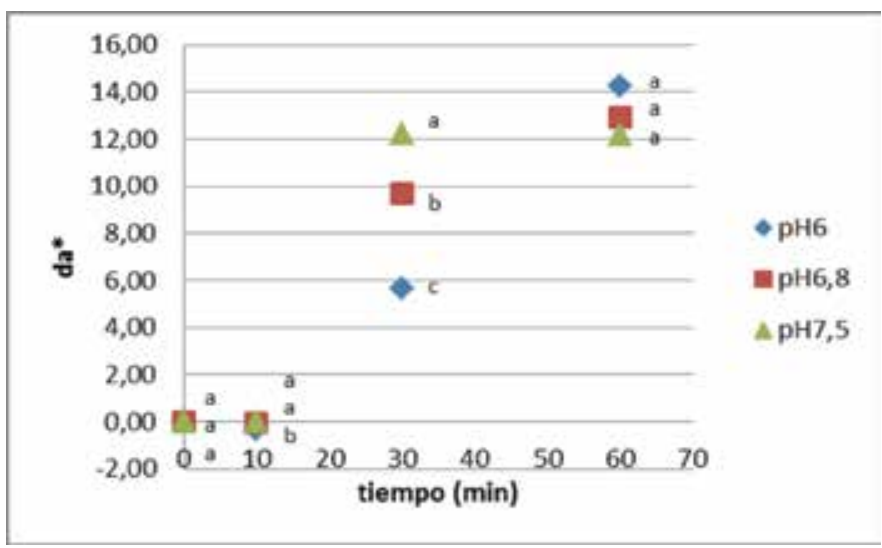

Figura 4. Diferencia del parámetro del color $a^{*}$ vs. tiempo de tratamiento térmico para los $3 \mathrm{pH}$ iniciales (medias con una letra común no son significativamente diferentes para cada tiempo, $\alpha \leq 0,05)$. 
El parámetro del color b* (Figura 5) aumentó en el tiempo de tratamiento térmico (colores más amarillos) con valores mayores a mayor $\mathrm{pH}$ inicial. Por el contrario, al tiempo final $\mathrm{db}^{*}$ disminuye (colores menos amarillos) para los $\mathrm{pH}$ iniciales 6,8 y 7,5, y es menor a mayor $\mathrm{pH}$ inicial.

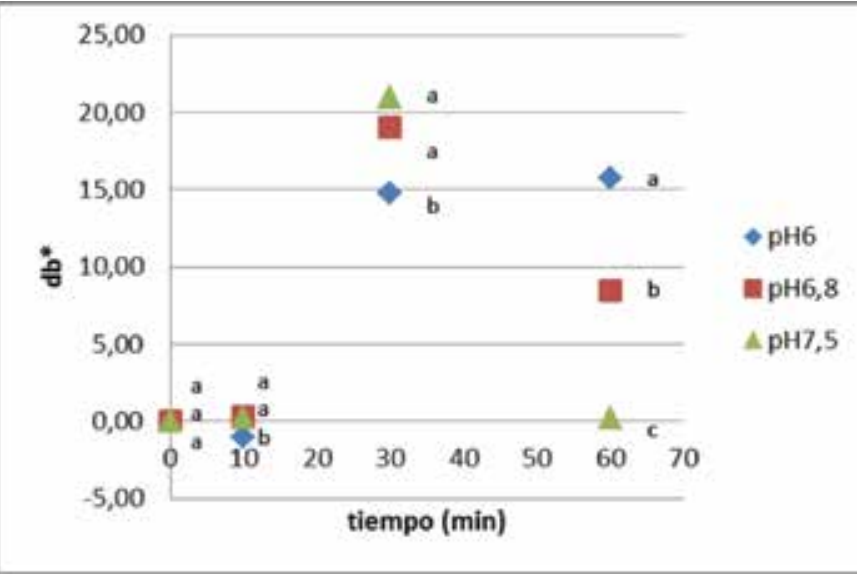

Figura 5. Diferencia del parámetro del color $b^{*}$ vs. tiempo de tratamiento térmico para los $3 \mathrm{pH}$ iniciales (medias con una letra común no son significativamente diferentes para cada tiempo, $\alpha \leq 0,05)$.

Ambas metodologías, K/S y dE*ab permitieron evaluar en forma cuantitativa diferencias de color entre las muestras con distintos tratamientos ( $\mathrm{pH}$ inicial y tiempo de calentamiento). Con la metodología CIELAB se pueden evaluar, además, las diferencias desde el punto de vista de la cromaticidad $\left(a^{*}, b^{*}\right)$ y la luminosidad del color ( $\left.\mathrm{L}^{*}\right)$.

El uso de estas metodologías se podría extender a la evaluación del color en muestras respecto a una muestra patrón, lo cual tendría gran aplicación en control de calidad e inclusive como herramienta en desarrollo, estandarización y/o caracterización de este tipo de productos.

\section{Conclusiones}

Las diferencias en los parámetros del color investigados, en función del tiempo de reacción y el pH, permitieron demostrar que los pigmentos coloreados formados en las reacciones de Maillard en las condiciones estudiadas fueron diferentes y aumentaron con el tiempo y el $\mathrm{pH}$.

La tendencia del color durante el calentamiento fue hacia el aumento del componente rojo y del color pardo global, y a la disminución de la luminosidad.

Las diferencias de color respecto al $\mathrm{pH}$ y tiempo presentaron cambios en la luminosidad y la cromaticidad $\left(\mathrm{a}^{*} \mathrm{y} \mathrm{b}^{*}\right)$, que se pudieron observar con ambos parámetros ensayados $(\mathrm{K} / \mathrm{S}$ y dE*ab).

Los parámetros estudiados, principalmente el índice $\mathrm{K} / \mathrm{S}$, serían muy adecuados para el seguimiento de la cinética de desarrollo del color $\mathrm{y}$, por ende, para un control adecuado de la calidad final del producto y el diseño de equipos específicos utilizados en la industria del dulce de leche $\mathrm{y}$ de otros productos lácteos concentrados $\mathrm{y}$ azucarados.

\section{Reconocimientos}

Por el soporte financiero a PEDECIBA, CSIC y LATU. Por la donación del caseinato de sodio a ERESUR S.A.

\section{Referencias}

- ASHOOR, S. H.; ZENT, J. B. Maillard browning in common amino acids and sugars. En: Journal of Food Science. 1984, 49:1206-1207.

- BADUI, S. D. Química de los alimentos. 3ra ed. México: Addison Wesley Longman de México, 1999.

- BUERA, M. del P.; HOUGH, G.; MARTINEZ, E.; RESNIK, S. Colorimetric, spectophotometric and sensory color measurements of a dairy product: dulce de leche. En: Anales de la Asociación de Química Argentina. 1990, 78(5):291-295.

- CALVO, C.; DURÁN, L. Propiedades físicas II. Ópticas y color. En: AGUILERA, J.M. Temas en tecnología de alimentos. Vol 1. México: Instituto Politécnico Nacional, 1997.

- CASTAÑEDA, R.; MUSET, G.; CASTELLS, L.; ARANIBAR, G.; MURPHY, M.; RODRÍGUEZ, G. Dulce de leche argentino variedad tradicional. Su caracterización. En: INTI. 5ta Jornadas de Desarrollo e Innovación. Buenos Aires: INTI, 2004.

- DATTATREYA, A.; RANKIN, S.A. Moderately acidic $\mathrm{pH}$ potentiates browning of sweet whey powder. En: International Dairy Journal. 2006, 16: 822-828.

- DAVIES, C.G.A.; LABUZA, T.P. The Maillard reaction application to confectionary products. Minnesota: University of Minnesota. Department of Food Science and Nutrition, 2003. pp. 1-33.

- DELGADO, T. Informe de tesis: contenido en furosina, lactulosa y $\beta$-lactoglobulina como indicadores de calidad en leche líquida y en polvo. Madrid: Universidad Complutense de Madrid. Instituto de Fermentaciones Industriales, 1993.

- DEMIATE, I.M.; KONKE, F.E.; PEDROSO, R.A. Avaliaçao da qualidade de amostras comerciais de doce de leite pastoso, composiçao química. En: Ciência e Tecnologia de Alimentos. 2001, 21(1):108-114.

- FAYLE, S.E.; GERARD, J.A. La reacción de Maillard. Zaragoza: Acribia, 2005

- FERRER, E.; ALEGRÍA, A.; FARRÉ, R.; ABELLÁN, P.; ROMERO, F. Indicadores del deterioro de la calidad proteica y del valor nutritivo de la leche. En: Food Science and Technology International. 1999, 5(6): 447-461.

- HAYASHI, T.; NAMIKI, M. Role of sugar fragmentation in an early stage browning of amino-carbonyl reaction of sugar with amino acid En: Agricultural and Biological Chemistry. 1986, 50(8):1965-1970.

- HAYASHI, T.; NAMIKI, M. Role of sugar fragmentation in the Maillard reaction. En: FUJIMAKI, M.; NAMIKI, M.; KATO, H. Susono. AminoCarbonyl reactions in food and biological systems. Tokio: Elsevier, 1986.

- HOUGH, G.; CONTARINI, A.; MORO O. Análisis sensorial de preferencia en dulce de leche. En: La Alimentación Latinoamericana. 1986, 20:72-74

- HOUGH, G.; BUERA, M.P.; MARTINEZ, E.; RESNIK, S. Effect of composition on nonenzymatic browning rate in Dulce de Leche like systems. En: An. Asoc. Quim. Arg. 1991, 79:31-34.

- HOUGH, G; BRATCHELL, N; WAKELING, I. Consumer preference of dulce de leche among students in the United Kingdom. En: J. Sensory Studies. 1992, 7:119-132.

- JIMÉNEZ, A.R.; HERNÁNDEZ, G.E.; VILLANOVA B.G. Evolution of non-enzymatic browning during storage of infant rice cereal. En: Food Chemistry. 2003, 83(2):219-225.

- KATO, H.; YAMAMOTO, M.; FUJIMAKI, M. Mechanisms of browning degradation of $\mathrm{D}$-fructose in special comparison with Dglucose- glycine reaction. En: Agricultural and Biological Chemistry. 1969, 33:939.

- MALEC, L.S.; LLOSA, R.A.; VIGO, M.S. Sugar formulation effect on available lysine content of dulce de leche. En: Journal of Dairy Research. 1999, 66(2):335-339.

- NURSTEN, Harry. The maillard reaction chemistry, biochemistry and implications. London: The Royal Society of Chemistry, 2005.

- PAULETTI, M.S.; MATTA, E.J.; ROZYCKI, S. Kinetics of heatinduced browning in concentrated milk with sucrose as affected by $\mathrm{pH}$ and temperature. En: Food Science and Technology International. 1999, 5(5):407-413.

- PAULETTI, M.S. Fabricación de Dulce de Leche. En: PÉREZ ÁLVAREZ, J.A.; FERNÁNDEZ LÓPEZ, J.;SAYAS BARBERÁS, E. Industrialización de productos de origen animal. Alicante: Universidad Manuel Hernández, 2001. pp. 255-280. ISBN 84-95315-65-3. 
- PETRIELLA, C.; RESNIK, S.L.; LOZANO, R.D.; CHIRIFE, J. Kinetics of deteriorative reactions in model food systems of high water activity: color changes due to nonenzymatic browning. En: Journal of Food Science. 1985, 50(3):622-626.

- ROZYCKI, S.D. Browning kinetics in concentrated dairy systems: comparative analysis of models. En: Ciencia y Tecnología Alimentaria. 2003, 4(2):95-102.

- ROZYCKI , S. D.; PAUlETTI, M. S.; COSTA, S. C.; PIAGENTINI, A. M.; BUERA, M. P. The kinetics of colour and fluorescence development in concentrated milk systems. En: International Dairy Journal. 2007, 17(8):907-915.

- ROZYCKI, S.D.; BUERA, M.P.; PAULETTI, M.S. Heat-induced changes in dairy products containing sucrose. En: Food Chemistry. 2010, (118):67-73

- TAMIME A. Y. Dairy powders and concentrated milk products. Singapur: Whiley and Sons, 2009.

- WROLSTAD, R.E.; SMITH, D.E. Color analysis. En: NIELSEN, S.S. Food analysis. 4a ed. New York: Springer, 2010.

- WOLFROM, M. L.; KASHIMURA, N.; HORTON, D. Factors affecting the Maillard browning reaction between sugars and amino acids. Studies on the non-enzymatic browning of dehydrated orange juice. En: Journal of Agricultural and Food Chemistry. 1974, 22:796-800. 